\title{
Les effets de la mixité au prisme du corps et de la sexualité
}

Les hommes dans les métiers d'accueil

The effects of gender diversity through the prism of the body and sexuality: men in receptionist jobs

\section{Sophie Louey et Gabrielle Schütz}

\section{OpenEdition}

\section{Journals}

Édition électronique

URL : http://journals.openedition.org/travailemploi/6466

DOI : $10.4000 /$ travailemploi.6466

ISSN : 1775-416X

\section{Éditeur}

DARES - Ministère du Travail

\section{Édition imprimée}

Date de publication : 1 octobre 2014

Pagination : 5-19

ISSN : 0224-4365

\section{Référence électronique}

Sophie Louey et Gabrielle Schütz, "Les effets de la mixité au prisme du corps et de la sexualité », Travail et Emploi [En ligne], 140 | octobre-décembre 2014, mis en ligne le 01 octobre 2016, consulté le 20 avril 2019. URL : http://journals.openedition.org/travailemploi/6466 ; DOI : 10.4000/ travailemploi.6466 


\title{
Les effets de la mixité au prisme du corps et de la sexualité : les hommes dans les métiers d'accueil $\left.{ }^{*}\right)$
}

\author{
Sophie Louey ${ }^{(* *)}$, Gabrielle Schütz ${ }^{(* *)}$
}

Les effets de la mixité sur la division sexuelle du travail et sur les carrières ont été peu étudiés lorsqu'elle résulte de la présence d'hommes dans des métiers dits "féminins». Cet article se propose de les explorer à partir du cas des hôte.sse.s d'accueil et en mettant l'accent sur la manière dont le corps et la sexualité, particulièrement impliqués dans cette activité, jouent sur les rapports de genre à l'euvre. Les deux enquêtes ethnographiques menées dans des situations d'accueil diversifiées montrent que les conceptions genrées du corps participent à la mise en place d'une division sexuelle des tâches, qui crée plus de latitude d'action et moins de contrôle pour les hommes dans l'activité quotidienne. La "drague» a des effets différenciés sur le personnel d'accueil. Elle concourt à préserver une définition traditionnelle et hétérosexuelle de la virilité des hôtes d'accueil, tout en leur permettant de sortir de la subordination et de la marginalité dérivant de leur position dans la division du travail et de l'emploi. À l'inverse, elle maintient les hôtesses d'accueil dans un certain isolement professionnel. L'étude des carrières du personnel d'accueil permet enfin d'évaluer l'existence d'un éventuel « escalator de verre» pour les hommes.

Les travaux sur la mixité ont montré que celleci a des conséquences ambivalentes, puisqu'elle produit à la fois une perpétuation de rapports inégalitaires entre les sexes et un renouveau de leur expression (Baudoux, Zaidman, 1992; CacouaultBitaud, Charles, 2011). Dans la sphère productive en particulier, elle se traduit par une recréation continue d'espaces ségrégués, déplaçant sans cesse les frontières de la division sexuelle du travail (Fortino, 1999, 2002). Les enjeux de la mixité au travail ont été beaucoup étudiés du point de vue de l'arrivée de femmes dans des bastions masculins. L'accès des femmes à ces métiers n'a pas abouti à une égalité des tâches dans l'exercice quotidien, ni à une égalisation des carrières, les femmes se heurtant à un «plafond de verre» au fur et à mesure qu'elles s'élèvent dans la hiérarchie (LAUFER, 2005; Guillaume, Pochic, 2007 ; Buscatto, Marry, 2009). En revanche, les situations d' «inversion du genre» où des hommes investissent des professions «féminines» ont été moins analysées (WILLIAMS, 1992; Angeloff, Arborio, 2002; Guichard-Claudic et

(*) Nous remercions les membres du laboratoire du Curapp (Centre universitaire de recherches sur l'action publique et le politique) ainsi que Stéphanie Guyon, Isabelle Clair et Fabrice Guilbaud pour avoir discuté ce texte.

(**) Université de Picardie, laboratoire Curapp; sophie. louey@u-picardie.fr

$(* * *)$ Université de Versailles - Saint-Quentin-en-Yvelynes, laboratoire Printemps (Professions, institutions, temporalités); gabrielle.schutz@uvsq.fr al., 2008; Buscatto, Fusulier, 2013). Ces travaux sur des hommes en situation de «minorité» se sont essentiellement concentrés sur les secteurs de la santé (Kergoat, 1992; Charrier, 2004 et 2008; Douguet, Vilbrod, 2008; Рicot, 2011), du travail social (Philippe, 2008; Bessin, 2008; Cresson, 2010; Bodin, 2011) et du champ éducatif (Galbraith, 1992; Murcier, 2008; Herman, 2007; JABOIN, 2008), voire sur les trois à la fois (WILLIAMS, 1992; Cross, Bagilhole, 2002)(1). Ils ont montré que l'avancée en mixité se traduit par des processus différents lorsqu'elle est le fait d'hommes et non de femmes, puisque leurs modalités d'entrée dans la profession où ils/elles sont «minoritaires», mais aussi le prix à payer pour cette position et leurs déroulements de carrières (GUICHARD-ClaUdiC et al., 2008) sont contrastés. Ces études illustrent comment l'«idéologie de la complémentarité» (HERMAN, 2007) produit des assignations de genre dans l'exercice de l'activité; elles font généralement état de l'existence d'un «escalator de verre» (Williams, 1992), métaphore qui fait référence aux mécanismes qui profitent aux hommes occupant des professions «féminines», et les conduisent à être intégrés plutôt facilement et à bénéficier rapidement de promotions.

(1) De manière beaucoup plus isolée, Pierre-Emmanuel SORIGNET (2004) a exploré l'«inversion du genre» dans un domaine artistique «féminin»: la danse. 
Notre étude entend ainsi apporter une contribution à l'analyse des situations professionnelles où les hommes font figure d'exception au sein de métiers dits "féminins», à partir d'un emploi de service appartenant à un secteur d'activité relativement peu exploré par cette littérature : celui des services marchands (2). Partir des hôte.sse.s d'accueil (voir encadré 1) permet d'aborder, de manière quelque peu renouvelée, la façon dont se jouent les rapports de genre dans ces situations. En effet, dans un métier de représentation comme l'accueil, le corps en tant qu'outil de séduction se trouve fortement mis à contribution et cristallise dès lors des enjeux liés à la définition de la féminité comme de la masculinité. Le «jeu des apparences» (HIDRI NeYs, 2013) constitue une trame de fond de cet emploi de service.

La question d'éventuelles renégociations de l'identité masculine dans ces activités «féminines », dont on sait qu'elles exposent les hommes au soupçon d'homosexualité (Cross, Bagilhole, 2002), s'y trouve posée de manière particulière. Une analyse attentive à la «routine relationnelle» (BROCHIER, 2011) de l'accueil permet de mettre plus spécifiquement l'accent sur le rôle joué par le corps, mais aussi par la sexualité, dans la manière dont s'élaborent dans ces situations une division sexuelle du travail et des avancées de carrière différenciées des un.e.s et des autres. Les études sur la sexualité dans les organisations ont généralement pointé le fait que celle-ci contribue à maintenir la subordination des femmes, en insistant en particulier sur le rôle du harcèlement (MacKInNON, 1979; Cromer, Lemaire, 2007). Réciproquement, partir d'une situation professionnelle comme l'accueil, où les hommes sont «minoritaires », permet d'aborder plus précisément la façon dont s'articulent sexualité dans les organisations, division du travail entre hommes et femmes et effets sur les carrières masculines.

Nous nous pencherons tout d'abord sur la question de la division sexuelle du travail entre hôtes et hôtesses d'accueil. Au-delà d'une mise en évidence de tâches différenciées, l'analyse montrera que la mixité à l'accueil crée plus de liberté pour les hommes dans l'exercice de leur activité. Étudiant ensuite comment le corps et la sexualité des hôtes et des hôtesses sont mis en jeu dans l'accueil, nous nous concentrerons sur le quotidien des interactions et, en particulier, sur les effets de la «drague» sur le personnel d'accueil. Enfin, l'analyse portera sur les carrières des un.e.s et des autres, et interrogera la pertinence de la notion d' «escalator de verre» dans ce contexte.

(2) À quelques exceptions près, comme Henri Peretz (1992) sur la vente de prêt-à-porter de luxe, Kevin D. Henson et Jackie K. Rogers (2001) sur le secrétariat en intérim, Emmanuelle LADA (2008) sur les agents d'accueil d'une entreprise publique de transport, Sophie BERNARD (2011) sur les caissiers.

\section{Hôtes et hôtesses au travail : l'inégalité dans la mixité}

Si des hommes et des femmes sont employés comme hôte.sse.s d'accueil, cela ne signifie pas pour autant que les un.e.s et les autres exercent les mêmes activités. Hôtes et hôtesses n'effectuent pas les mêmes tâches, qu'ils occupent ou non les mêmes postes, et ont généralement intériorisé ce partage, qu'ils participent à justifier et à pérenniser.

Tout d'abord, certaines missions d'accueil sont exclusivement confiées à des hommes. En accueil événementiel par exemple, si les femmes peuvent être embauchées comme chauffeuses pour conduire les «VIP» (Very Important Person) à une réception, seuls des hommes sont engagés comme voituriers pour garer les véhicules des invités lors d'une soirée. Traditionnellement, les missions d'accueil dans les défilés de mode sont également réservées aux seuls hommes - nous y reviendrons - et celles d'hommesandwich ne se déclinent pas non plus au féminin. Ensuite, lorsqu'ils opèrent au sein d'une même mission d'accueil, hommes et femmes ne sont pas pour autant coprésents : ils sont souvent affectés à des postes différents ou aux mêmes postes mais à des horaires distincts. En accueil en entreprise ainsi, lorsqu'une société ferme particulièrement tard le soir, elle peut pour cette tranche horaire préférer un homme à son accueil, plus facilement confondu avec un agent de sécurité et donc pensé plus dissuasif. Lorsqu'une même société comporte plusieurs accueils, hommes et femmes ne se répartissent pas non plus au hasard. Selon un des enquêtés, Marc, 36 ans et hôte à temps plein depuis six ans ${ }^{(3)}$, le poste d'accueil du dernier étage de la grande société de conseil pour laquelle il travaille en tant que prestataire, est par exemple systématiquement attribué à une femme : il s'agit d'un accueil éminemment protocolaire où la tâche principale consiste à recevoir les visiteurs de la direction. Son propre poste, en revanche, a toujours été occupé par un homme : celui-ci s'apparente d'ailleurs plus à une conciergerie qu'à un accueil, puisqu'il s'agit de centraliser et répartir les demandes d'intervention des salariés qui ont trait à la maintenance ou à la propreté, et d'organiser pour eux des services à la personne (service de pressing, de tirage de photos, etc.). Les hôtes sont ainsi rarement affectés à des postes de seule représentation. On constate d'ailleurs, en entreprise, que l'on trouve très peu d'hôtes sur des accueils «purs»: ils opèrent sur des accueils/standards et plus souvent encore sur des standards simples. De la même manière, en accueil événementiel, on voit rarement des hôtes d'accueil immobiles debout les mains dans le dos, chargés de sourire à toute personne passant devant eux, ou encore disposés

(3) Pour en savoir plus sur chaque enquêté, nous renvoyons au tableau en annexe; entretien du 7 janvier 2007. 


\section{Encadré 1 \\ Deux enquêtes sur les hôte.sse.s d'accueil}

Accueillir constitue ce que Michelle PERROT (1987) appelle un «métier de femme». Correspondant généralement à des emplois à temps partiel, l'accueil est souvent présenté comme permettant la «conciliation " entre vie professionnelle et vie domestique, et s'inscrit pleinement dans une division sexuelle du travail qui réserve aux femmes les tâches d'assistance et de représentation. De fait, l'accueil est très majoritairement exercé par des femmes et requiert des compétences pensées comme "féminines" (accueillir, materner, faire preuve de diplomatie), tandis qu'il impose une tenue et une présentation de soi, elles aussi, associées à un imaginaire "féminin " (discrétion, élégance, séduction).

L'accueil peut être exercé selon diverses modalités. L'accueil dit «événementiel» s'effectue en contrat à durée déterminée (CDD) et très majoritairement via des prestataires de services, qui embauchent et habillent leur personnel pour accueillir et orienter les visiteurs des manifestations organisées par leurs clients (salons grand public ou professionnels, congrès et conventions d'entreprise, événements sportifs, soirées et cocktails). Les prestataires recrutent de manière permanente, afin de constituer un vivier dans lequel puiser au gré des missions; ils mettent en place généralement autant de sessions de recrutement pour les hommes que pour les femmes, même si ces dernières sont plus nombreuses à postuler et sont sollicitées pour un nombre beaucoup plus élevé de missions. La grande majorité du personnel d'accueil en événementiel exerce cette activité de manière complémentaire à une autre : hôtes et hôtesses sont étudiants, parfois en recherche d'un premier emploi après avoir achevé leurs études, ou encore se consacrent à une activité artistique dont ils ne parviennent pas à vivre (ScHÜTZ, 2011).

L'accueil «en entreprise" s'exerce, quant à lui, en contrat à durée indéterminée (CDI), très souvent à temps partiel. II consiste principalement à accueillir et orienter les visiteurs d'une entreprise depuis une borne d'accueil située dans le hall d'entrée, qui sert parfois simultanément de standard téléphonique. Les hôtesses ${ }^{(1)}$ peuvent ainsi faire uniquement de l'accueil dit «physique» ou répondre en plus au téléphone et effectuer dès lors un accueil dit "mixte»; ou encore, plus rarement, être simplement standardistes, auquel cas elles travaillent dans des locaux spécifiques et non à l'entrée. Comme nous l'avons observé, ces hôtesses en entreprise sont moins souvent étudiantes que les hôtesses de l'événementiel et un peu plus âgées; elles sont généralement issues de milieux plus populaires et font de cet emploi leur activité principale pour près d'un tiers d'entre elles - les autres recherchent un emploi dans une autre branche ou sont étudiantes, plus rarement artistes. L'externalisation de l'accueil en entreprise gagne du terrain depuis le milieu des années 1990 : on peut aujourd'hui estimer qu'environ deux tiers des entreprises de plus de 250 salariés confient leur accueil à un prestataire - toutefois, nombre de petites et moyennes entreprises (PME) externalisent également leur accueil, et le recours à cette prestation concerne l'ensemble des secteurs d'activité, le privé comme le public, administrations comprises (SchüTz, 2011). L'enquête menée sur les prestataires de services d'accueil permet d'estimer la proportion d'hôtes d'accueil en entreprise entre 5 et $10 \%$ au maximum. Nous ne disposons pas de chiffres, en revanche, pour les accueils non externalisés.

Les résultats présentés dans cet article proviennent de deux enquêtes distinctes. Gabrielle Schütz a enquêté sur les prestataires de services d'hôtesses d'accueil. Elle a réalisé des observations comme hôtesse d'accueil en événementiel sur une quinzaine de missions en CDD, d'une durée variant de quelques heures à trois semaines, puis a effectué deux stages au siège de deux sociétés prestataires parisiennes, l'une de taille moyenne, Angélique Hôtesses (2), l'autre de très grande taille et disposant d'antennes sur tout le territoire, Business Accueil. Ces stages ont respectivement duré six et deux mois; ils lui ont permis d'observer le travail des responsables de l'accueil en entreprise de ces sociétés, mais aussi de se rendre sur une quinzaine de bornes d'accueil de différentes entreprises clientes, d'y passer un à deux jours à chaque fois et d'y observer une vingtaine d'hôtesses en situation de travail. Des entretiens avec du personnel de ces deux sociétés et d'autres prestataires d'accueil (parmi lesquels Facil'Otess, Bienvenue et Réceptor) sont venus compléter le dispositif d'observation : avec des hôte.sse.s en événementiel et en accueil en entreprise $(n=37)$, avec des salariés responsables de ces prestations travaillant au siège des sociétés prestataires $(n=29)$. Enfin, des entretiens avec des clients des deux types de prestations ont été réalisés $(n=14)$. Une sociographie des hôte.sse.s a été ébauchée à partir de l'exploitation d'une base de données, créée à partir des dossiers du personnel d'Angélique Hôtesses. La base comporte 279 hôtesses en accueil en entreprise, 246 hôtesses et 134 hôtes en accueil événementiel. Enfin, plusieurs corpus documentaires ont été analysés, parmi lesquels des revues professionnelles.

Sophie Louey a effectué, quant à elle, une monographie au sein de l'équipe d'accueil d'Entreprendre, une entreprise de type parapublic située dans le nord de la France, qui compte une soixantaine de salariés auxquels s'ajoute une quinzaine de locataires de bureaux, qui sont physiquement présents dans le même bâtiment. Occupant un poste d'hôtesse d'accueil à temps partiel sous contrat étudiant pendant trois années consécutives, elle a rejoint une équipe composée de quatre autres personnes, toutes en CDI et parmi lesquelles, deux «statutaires» (une femme à temps partiel et un homme à temps plein) et deux "contrats étudiants" (une femme et un homme à temps partiel). Les hôte.sse.s exercent par équipe de deux. Ces équipes ne sont pas fixes : elles peuvent tantôt être mixtes ou être constituées uniquement de femmes ou d'hommes. L'ensemble des hôte.sse.s est rarement rassemblé, si ce n'est à l'occasion des repas de Noël ou encore d'anniversaires. À l'accueil physique et téléphonique s'ajoutent pour le personnel des tâches administratives, telles que les mises sous plis et l'affranchissement du courrier de l'entreprise, la gestion des réservations des véhicules pour les employés, celles des salles du bâtiment, etc. Des entretiens ont été effectués avec chaque membre de l'équipe d'accueil ainsi qu'avec des employé.e.s d'autres services; une observation prolongée des interactions entre hôte.sse.s, mais aussi entre les hôte.sse.s et les autres salarié.e.s de l'entreprise ou les individus extérieurs s'adressant à l'accueil, a également été réalisée.

(1) Ce sont en très grande majorité des femmes, $c f$. infra.

(2) Tous les noms propres cités dans l'article ont été modifiés. 
en «haie d'honneur» sur le passage des visiteurs comme c'est souvent le lot des hôtesses. Plutôt qu'à des postes à visée décorative, les hôtes sont préférés pour ceux impliquant du filtrage et de la gestion de flux : on les rencontre par exemple dans les parkings d'un lieu de réception, où ils doivent indiquer aux visiteurs où se garer, ou au placement des invités.

Il arrive cependant qu'hôtes et hôtesses se côtoient à un même accueil auquel ils sont affectés de manière a priori interchangeable. L'observation à Entreprendre montre toutefois que même en ce cas des différences se recréent. Plus précisément, lorsque l'hôte et l'hôtesse exercent séparément, les tâches effectuées au travail sont globalement les mêmes. En revanche dès qu'ils exercent «ensemble», c'est-à-dire en binôme, des différences apparaissent, en premier lieu parce que les demandes qui leur sont adressées ne sont pas les mêmes. Par exemple, plutôt que les hôtesses, ce sont les hôtes qui sont systématiquement sollicités pour les tâches impromptues exigeant une action physique (telles que déplacer une table). À l'inverse, les activités quotidiennes dites de «petites mains», qui s'accomplissent derrière le guichet (mises sous plis, affranchissement du courrier), sont attribuées aux hôtesses alors même que, contractuellement, ce type de tâche administrative revient tout autant aux hôtes. Si certaines demandes s'adressent préférentiellement aux hôtes ou aux hôtesses, au sein de l'équipe d'accueil, les tâches tendent néanmoins à être en partie redistribuées. Les hôtes font ainsi appel aux hôtesses pour les aider à déplacer une table plutôt qu'à des collègues masculins à des postes hiérarchiques supérieurs; les hôtesses se tournent, quant à elles, vers les hôtes pour partager avec eux la charge de travail administratif qu' on leur confie. C'est donc une logique de statut qui prévaut au sein des équipes d'accueil plus qu'une logique de genre. Néanmoins, les distinctions entre tâches des hôtes et des hôtesses ne disparaissent pas tout à fait avec ces arrangements internes à l'accueil, tant les représentations genrées sont fortes et incorporées, comme le montrent les propos du personnel de Entreprendre :

"On fait des trucs de mecs; si on n'était pas là, ce serait plus compliqué pour [les hôtesses]. Des fois, on porte des trucs lourds; on peut aussi être vachement importants quand il y a des visiteurs difficiles, parce qu'un mec, ça a quand même plus d'autorité, il faut le dire! »

(Christophe, 30 ans, hôte d'accueil à temps plein à Entreprendre, depuis 5 ans; entretien du 4 novembre 2011.)

«Les filles, on a plus les trucs de mises sous plis, tout ça, alors que les mecs, c'est plus du genre de monter les colis dans les services. [...] Après, on fait le même boulot quoi; on accueille, on répond au téléphone, donc il n'y a pas de différences, on fait tous la même chose. [...] Parfois les mecs, ils en ont marre de mettre sous enveloppes ou de préparer des dossiers, alors on le fait. On a peut-être plus de patience et on est plus méthodiques.»

(Pauline, 22 ans, hôtesse d'accueil à temps partiel à Entreprendre, depuis deux ans et demi; entretien du 23 décembre 2011.)

La mixité de l'accueil ne se traduit donc ni par une «inversion du genre» (GuICHARD-Claudic et al., 2008) ni même par une égalité des tâches. Au contraire, se reconstruisent en son sein des tâches pensées comme «féminines» ou «masculines». Celles-ci sont marquées par des stéréotypes de genre qui attribuent aux hôtes la force, l'initiative, l'autorité, mais aussi les tâches «techniques» ou se déroulant à l'extérieur des accueils à proprement parler tandis qu'aux hôtesses, sont attribuées la minutie, les missions de représentation, la passivité, les tâches en intérieur et le "travail décoratif ${ }^{(4)}$ ». $\mathrm{Si}$ les hôtesses mettent spontanément en avant une égalité de tâches avec les hôtes, ces derniers insistent plutôt, quant à eux - comme les recruteurs (cf. infra) -, sur le «plus» que leur présence apporterait, comme l'ont montré les précédents extraits d'entretiens ${ }^{(5)}$. Ces constats font écho aux travaux féministes qui, comme celui de Danièle KERGOAT (2000, p. 36), ont montré que derrière la division du travail entre hommes et femmes se jouent non seulement un principe de séparation des tâches mais aussi leur hiérarchisation et une valeur sociale différente accordée au travail de chaque sexe.

Même si les hommes se voient confier certaines tâches pénibles physiquement - qu'ils n'hésitent pas à partager avec leurs collègues féminines - il apparaît que la répartition sexuée du travail produit bel et bien pour eux un «plus» en termes d'autonomie et d'initiative dans leur labeur quotidien. Moins astreints au travail de représentation et au travail décoratif, parvenant à éviter partiellement les tâches administratives les plus répétitives, les hôtes ont plus d'opportunités de s'échapper de l'espace cloisonné de l'accueil, qui astreint dès lors physiquement davantage les hôtesses. À cet égard, les différences entre hôtes et hôtesses rejouent ce qui s'observe dans d'autres espaces productifs : Michel Gollac et Serge Volkoff (2002) ont ainsi montré comment les ouvriers bénéficient d'une mobilité spatiale et d'un champ d'action bien moins restreints

(4) Barbara GUTEK (1995) définit comme «decorative work» les emplois où être beau et attrayant - plus fréquemment, belle et attrayante - constitue une part primordiale du travail.

(5) Au-delà de l'autocongratulation, ces discours des hôtes peuvent aussi être lus comme une justification de la transgression des frontières du genre, qui les mène à occuper une fonction généralement dévolue aux femmes. On retrouve chez d'autres hommes dans des professions «féminines» des propos similaires ( $c f$. entre autres Cross, BAGILHOLE, 2002; ChARRIER, 2004; JABOIN, 2008; Bessin, 2008), qui font écho à la manière dont certaines femmes se justifient d'exercer dans des métiers d'hommes par le «plus» qu'apportent leurs qualités «féminines », à l'instar de femmes agents de sécurité (BAUVET, 2010) ou cadres (LAUFER, 1982). 
que les ouvrières; ils travaillent par exemple beaucoup moins souvent à la chaîne.

\section{Féminité et virilité à l'accueil : des usages du corps et de la "drague"}

Cet inégal arrimage des hôtes et des hôtesses à l'accueil a des conséquences sur les usages et le contrôle du corps des un.e.s et des autres; il se répercute aussi dans leurs relations aux visiteurs et aux autres salarié.e.s.

\section{Le genre des corps disciplinés}

L'accueil constitue, aux dires des prestataires et de leurs clients, une «vitrine» de l'entreprise, et fait peser à ce titre de nombreuses prescriptions corporelles sur le personnel, qui se doit d'avoir une «bonne présentation» et une «tenue correcte», tant du point de vue vestimentaire que de celui du maintien corporel (SchÜтZ, 2006). Néanmoins, ces prescriptions relativement génériques se distinguent par leur foisonnement en ce qui concerne les hôtesses. En plus de lister dans leur règlement tout ce que le personnel ne doit pas faire à un accueil(6), beaucoup de sociétés prestataires vont par exemple jusqu'à préciser la hauteur des talons des hôtesses, et à décrire avec force détails le type de collants, de bijoux ou encore de maquillage qu'elles doivent porter (ou ne pas porter), la manière dont leurs cheveux doivent être coiffés et attachés, voire la couleur exacte de leur rouge à lèvres et de leur vernis à ongles ${ }^{(7)}$. Ces prescriptions n'ont pas d'équivalent pour les hôtes d'accueil, auxquels il est simplement enjoint de porter un costume avec des chaussures cirées assorties, d'avoir les mains soignées, une coupe de cheveux nette et d'être rasé de près. La présentation des hôtes d'accueil est ainsi beaucoup moins codifiée que celle des hôtesses et fait l'objet d'une attention bien moindre ${ }^{(8)}$.

(6) Entre autres : être mal assis.e, mâcher un chewing-gum, manger devant les visiteurs, avoir le dos voûté, s'adosser ou s'asseoir quand l'accueil se fait debout.

(7) Ces prescriptions nous renseignent sur la place assignée aux hôtesses par la hiérarchie; elles ne nous disent toutefois rien des pratiques effectives. Certaines hôtesses «oublient» ainsi sciemment de s'attacher les cheveux ou de porter leur uniforme de travail, d'autres sont parfois jugées «vulgaires» par leur employeur ou par la société cliente : c'est le cas, par exemple, lorsqu'elles portent leurs propres habits ou lorsque leur maquillage semble inapproprié. Elles se font alors rappeler à l'ordre et réexpliciter de manière plus détaillée encore le type de féminité attendu d'elles.

(8) D’une manière générale, la marge d'improvisation vestimentaire dans la tenue de travail est beaucoup plus faible pour les hommes que pour les femmes. Aussi, cette codification plus grande pour les hôtesses doit-elle être également comprise car elles peuvent plus facilement que les hôtes dévier de la représentation de genre bourgeoise et policée attendue d'elles (SсHÜTZ, 2006).
Pesant préférentiellement sur les femmes, ces prescriptions physiques ont pour corollaire un contrôle plus acéré de l'apparence des hôtesses. En accueil événementiel, où les attentes corporelles sont portées à leur paroxysme, les hôtesses font parfois l'objet d'un passage en revue en début de mission, les «chefs hôtesses» scrutant la tenue, la coiffure et le maquillage de chacune d'entre elles avec minutie. Moins souvent affectées en extérieur et plus souvent rivées à leur poste, elles sont également plus visibles, et échappent par conséquent moins souvent à la surveillance de leurs supérieures. Elles sont ainsi régulièrement reprises sur leur maintien et leur attitude : "Tiens-toi droite!», «Ne tourne pas le dos!», "Souris!». Éléments d'un tableau d'ensemble, elles doivent bien souvent demander la permission pour s'absenter quelques minutes et se rendre aux toilettes. Plus libres de leurs mouvements, n'évoluant pas en jupe étroite et sur des talons hauts qui entravent leur mobilité, les hôtes sont pour leur part soumis à un contrôle corporel bien moindre ${ }^{(9)}$, comme le note cet hôte d'accueil en événementiel de la société prestataire Bienvenue :

«Les chefs hôtesses, par rapport aux hôtesses, sont beaucoup plus strictes avec elles que nous, nos chefs hôtes par rapport aux hôtes. Nous c'est plus... on est plus indépendants en fait, alors que les hôtesses, apparemment - je sais pas si c'est propre à Bienvenue - c'est vraiment plus strict, notamment sur les coiffures... tout, en fait. Les chefs hôtesses sont là, la ponctualité, la tenue des cheveux, enfin très strictes. Ça c'est une différence, et ça se remarque aussi sur les opérations mixtes justement, où justement tu te retrouves avec des chefs hôtesses, qui gèrent des garçons et des hôtesses... Mais moi, j'ai d'autres hôtesses avec qui j'ai bossé, qui m'ont fait la remarque: "Putain, vous les mecs, avec vous, elles sont beaucoup plus cool les chefs." Leurs chefs à elles, qui sont aussi nos chefs à nous, sont beaucoup plus coulantes avec nous qu'avec elles. »

(Nicolas, 25 ans, hôte d'accueil en événementiel à Bienvenue, depuis quatre ans et demi; entretien du 1 er juin 2004.).

En accueil en entreprise, les prescriptions corporelles qui pèsent sur le personnel d'accueil sont généralement moins contraignantes ${ }^{(10)}$. Un contrôle des corps ne s'en exerce pas moins sous des formes beaucoup plus diffuses, mais tout

(9) Selon Colette Guillaumin (1992, p. 86), en plus de rendre moins stables les femmes, le port de la jupe - qu'elle décrit comme un "volant autour du sexe, fixé à la taille comme un abat-jour» - les rend sexuellement disponibles en offrant leur corps et en les forçant à lui porter une attention accrue afin de le protéger.

(10) La sélection des hôte.sse.s de l'accueil en entreprise repose d'abord beaucoup moins sur des critères morphologiques, tels qu'une taille minimale et une corpulence limitée. Ensuite, le port de l'uniforme n'est pas systématisé, tandis que les corps, partiellement dissimulés derrière des guichets, sont aussi beaucoup moins exposés. 
aussi opérantes : il n'émane pas d'ordres impératifs de chefs hôtesses orchestrant un événement circonscrit dans le temps, mais est véhiculé plus discrètement par un ensemble beaucoup plus large de personnes, et agit dans la durée via la répétition. À Entreprendre, aucun uniforme n'est fourni au personnel d'accueil et la tenue de rigueur n'est pas explicitée, contrairement à ce qui se passe chez les prestataires d'accueil. Si hôtes et hôtesses se doivent d' «avoir une bonne présentation », comme c'est précisé dans leur contrat, les hôtesses sont dans les faits davantage soumises à cette obligation, qu'elles apprennent à retraduire en une injonction à être «féminines». Au quotidien, la tenue des hôtesses fait en effet l'objet de beaucoup plus d'attention que celle des hôtes, tant de la part de leur supérieure hiérarchique que de celle des autres salarié.e.s. Elles sont ainsi très régulièrement complimentées ou critiquées sur leur garde-robe, tandis que celle des hôtes est rarement commentée. Au fur et à mesure de leur intégration à Entreprendre, les deux hôtesses en contrat étudiant observées ont ainsi progressivement troqué leurs baskets et leurs vêtements décontractés contre des robes, des jupes et des chaussures à talons. Leur adaptation à leur environnement professionnel est ainsi passée par un travail sur leurs corps, qui les a menées à construire une image d'elles-mêmes conforme aux normes de désirabilité véhiculées par le regard hétérosexuel.

Non seulement le corps des hôtesses d'accueil fait l'objet d'un contrôle plus serré mais de plus, elles en intériorisent davantage la légitimité que les hôtes. À Entreprendre, alors que le personnel d'accueil n'a pas le droit de manger à la borne d'accueil, les hôtes se le permettent, tandis que les hôtesses s'isolent systématiquement pour «grignoter». Une des chefs hôtesses interviewées en accueil événementiel, Amina, souligne pour sa part que les hôtesses prennent leur mission de représentation beaucoup plus au sérieux que les hôtes :

"L'ambiance collective est beaucoup plus légère [quand il y a des hôtes], parce que généralement, les hôtes, ils sont là pour faire les... les... les clowns; enfin, ils font un peu... [...] Les critiques à faire [aux hôtes], déjà, c'est beaucoup moins... Enfin tu vois, y'a pas de maquillage, éventuellement il est pas rasé, mais ça, il le sait et il en est conscient généralement... [alors qu'une hôtesse n'a par exemple pas toujours conscience d'être "mal coiffée" ou "mal maquillée" selon les critères des prestataires, comme Amina l'a expliqué peu auparavant dans l'entretien] Bon, le costume, bon, tu vois mais bon... c'est pas la même chose, ils le prennent pas autant à cour aussi. Ils le prennent pas du tout autant à cœur, y'a une différence là-dessus. C'est pour ça je te dis que c'est beaucoup plus léger. [...] Après c'est vrai qu'ils sont moins gérables, sur certaines choses, parfois, tu vois, sur la... sur... sur tout ce qui est... [...] S'il y a une connerie à faire, ça va plus être eux tu vois, enfin c'est... Non, mais enfin voilà, vu qu'ils prennent moins au sérieux, plus à la légère, ils peuvent... je sais pas... faire... J'ai fait une mission en tant qu'hôtesse, pas en tant que chef hôtesse, y'a des hôtes qui se sont bourré la gueule sur une mission. »

(Amina, 25 ans, chef hôtesse événementiel pour Réceptor, depuis six ans; entretien du 25 novembre 2005.)

Les différences d'autocontrôle corporel entre hôtes et hôtesses ne renvoient pas qu'à une forme de «docilité féminine» favorisant l'apprentissage et le respect des règles. Plus qu'aux effets d'une docilité générique, cette relative acceptation des règles du jeu par les hôtesses relève plutôt d'une intériorisation genrée du contrôle corporel, qui se manifeste, comme le mentionne Amina, par le fait que les hôtesses prennent beaucoup plus «à cœur» les remarques que l'on peut leur faire sur leur apparence. Conformément en effet aux propos du critique d'art John Berger, cité par Rossella GHIGI (2004, p. 57) : «Les hommes regardent les femmes. Les femmes se regardent elles-mêmes en tant qu'objets de regards. Ceci détermine non seulement le rapport entre hommes et femmes, mais aussi le rapport des femmes avec elles-mêmes.» Les hôtesses, en tant que femmes, ont ainsi intériorisé la pression du regard masculin. Certaines peuvent être néanmoins critiques et s'irriter de la performance de genre attendue d'elles - le plus souvent celles pour qui cette activité est secondaire et transitoire, en particulier les étudiantes ou les jeunes femmes en recherche d'un autre emploi. D'autres jugent en revanche légitimes les contraintes corporelles qui pèsent sur elles, qu'elles justifient par leur mission d'accueil - le plus souvent celles qui font de cet emploi leur activité principale, dotées de moindres capitaux socio-économiques. Néanmoins, la plupart tâchent de s'y conformer tant qu'elles sont en poste. Pour les hôtes d'accueil en revanche, en tant qu'hommes, devoir prendre soin de leur apparence et jouer de celle-ci pour leur travail d'accueil, les place dans une posture quelque peu inconfortable. Leur identité de genre requiert d'eux de ne pas se prendre trop au sérieux dans ce rôle et donc, en particulier, de ne pas respecter scrupuleusement la règle ni de se formaliser des remarques qu'on pourrait leur faire, faute de quoi ils pourraient passer pour de peu virils personnages imbus de leur apparence, voire pour des homosexuels. L'enjeu d'être "la plus jolie», comme le recommandent certains prestataires à leurs hôtesses ${ }^{(11)}$, ne trouve pas d'équivalent chez les hôtes, être «le plus beau»

(11) «Votre maquillage ne doit surtout pas être agressif, mais l'on doit toujours sentir que vous avez pris le plus grand soin à être la plus jolie», précise ainsi la société prestataire Welcome dans sa «charte de qualité», que les hôtesses doivent signer en même temps que leur contrat. 
n'étant certainement pas un enjeu de la masculinité hégémonique (Connell, Messersschmidt, 2005) ${ }^{(12)}$.

Se disciplinant physiquement davantage que les hôtes, les hôtesses effectuent ce que Colette Guillaumin (1981, p. 25) nomme un «service corporel $^{(13)} \gg$. Cet usage du corps des hôtesses est, en dernière instance, lié au fait que leur rôle consiste à faire jouer l'imaginaire de la femme disponible et à laisser entrevoir une possibilité de contacts extraprofessionnels. Au-delà d'usages différenciés de leurs corps, ce sont donc les relations qu'ils entretiennent avec les personnes accueillies, comme avec les autres salarié.e.s, qui distinguent hôtes et hôtesses.

\section{La "drague" à l’accueil ou la recréation par-delà les statuts d'un entre-soi masculin}

S'interroger sur la drague à l'accueil permet de révéler les positions de chacun.e. En effet, les hôtesses subissent quotidiennement la drague des visiteurs, comme celle des salariés masculins des lieux sur lesquels elles travaillent, drague quasi rituelle au sens où il ne s'agit en général pas tant pour eux de tenter de les séduire que de réaffirmer publiquement leur identité masculine dans sa dimension hétérosexuée (SchüTz, 2006). Ainsi, les plaisanteries à caractère sexuel plus ou moins explicite adressées aux hôtesses par des hommes sont monnaie courante. Elles permettent aux hommes d'affirmer leur virilité, dont l'expression la plus légitime dans l'imaginaire commun est associée à une entreprenante hétérosexualité (ConNell, 1995). Face à ces discours, les hôtesses sont le plus souvent silencieuses, mais elles peuvent aussi occasionnellement faire preuve de grossièreté et se livrer à des «déviances langagières» (Zolesio, 2009), sortant ainsi de leur rôle d'accueil et de leur performance de genre $^{(14)}$.

Beaucoup plus que les autres femmes aux côtés desquelles elles peuvent être amenées à travailler, qui sont parfois tout aussi jeunes et jolies, les hôtesses ont ce statut d'objet des (tapageuses) convoitises et des plaisanteries grivoises. Ce statut

(12) La notion de «masculinité hégémonique», forgée par Robert W. ConNell $(1995,2005)$, désigne, par-delà les diverses incarnations de la masculinité, l'importance d'une norme, qui alors même qu'elle ne peut être assumée que par un nombre restreint d'hommes, constitue un idéal de référence - variable tant historiquement que géographiquement - et domine hiérarchiquement les autres formes de masculinité.

(13) Guillaumin (1981) utilise cette expression pour désigner ce que les femmes doivent aux hommes, mais aussi aux enfants ou encore aux malades, sans qu'elles bénéficient de contrepartie salariale. Dès lors, nous considérons le «service corporel» des femmes comme extensible au champ du travail salarié et aux travaux de représentation, dans la mesure où la plus grande discipline corporelle à laquelle doivent se soumettre les hôtesses, bien que prescrite, n'est pas reconnue.

(14) Ces formes de résistance, ponctuelles, ont lieu lors d'échanges restreints entre hôte.sse.s et, plus rarement, s'expriment parfois face à des visiteurs médusés. de cible de la drague est modulé en fonction du degré d'extériorité des hôtesses. Ainsi, parmi les hôtesses prestataires de l'accueil en entreprise, les «titulaires» d'un poste sont généralement moins sollicitées par les salariés hommes que les hôtesses «volantes», qui effectuent des remplacements ponctuels au pied levé. De même, lorsque les hôtesses sont toutes des salariées internes, comme à Entreprendre, parmi elles, les hôtesses dites «statutaires» sont moins exposées que les hôtesses avec des contrats "étudiants», dont la présence dans l'entreprise est plus brève et de fait moins assurée. La position hiérarchique des hôtesses - un des échelons les plus bas dans la plupart des organisations - comme leur statut salarial jouent donc dans le même sens, l'intensité de l'exposition à la drague allant de pair avec celle de la subordination. Ainsi, à Entreprendre, Laurence, ancienne hôtesse d'accueil en contrat étudiant pendant trois ans lors de ses études de droit, et devenue depuis chargée de mission, explique :

«J'étais une petite jeune, je me faisais souvent draguer. [...] Ça faisait partie de l'emploi. Aujourd'hui, je ne suis pas dans la réception; c'est moi qui mène les relations, j'ai un statut aujourd'hui qui fait qu'on me respecte $»$.

(Laurence, 39 ans, chargée de mission à Entreprendre; entretien du 25 octobre 2011.)

Fort différente est la situation des hôtes. Aucun cas d'hôte cible de drague n'a pu être directement observé lors des enquêtes; cependant, lors d'un entretien avec un hôte, des exemples ont été cités :

«Y'a un mec, il est carrément... il est venu voir trois potes. Moi, j'étais juste parti à ce moment-là. Il est venu les voir: "Vous avez des copines les gars?" et tout. Enfin, bien sûr, ils ont tous dit oui; en fait, ils ont tous dit oui. Et le gars auparavant, y'a un des hôtes, il lui avait demandé : "Ça te dirait de faire l'amour?" et tout, enfin... [...] Y'a un mec qui s'est fait tirer les poils d'ailleurs, du torse: "T'es trop sexy", et tout, enfin [il rit]! Oui, ça arrive... Y'a un mec, une fois, il a vraiment, il est carrément parti quoi; il est allé voir le chef hôte; les gars, ils le lâchaient pas : "Viens, on va en soirée" et tout!»

(Steve, 22 ans, hôte d'accueil en événementiel pour Bienvenue, depuis un an et demi; entretien du 17 mars 2004.)

Ces anecdotes se rapportent toutes à des cas de drague homosexuelle, et l'une d'entre elles avait pour cadre l'inauguration d'une boutique Yves-Saint-Laurent, où seuls des hôtes avaient été mobilisés, coiffés pour l'occasion comme le grand couturier et habillés d'un tailleur de la marque porté buste nu. De toutes les missions événementielles observées ou relatées, il s'agit de celle où la dimension décorative des hôtes d'accueil était la plus affirmée. Lorsque les hôtes sont cibles de drague, cela ne subvertit donc pas l'ordre genré, puisque cela se produit dans des contextes où ils sont pensés comme des objets esthétiques destinés 
à l'agrément d'autres hommes et non de femmes. Une des explications couramment donnée au fait que seuls des hôtes assurent l'accueil des défilés de mode, est en effet celle de la supposée prépondérance d'un public homosexuel masculin dans cet univers, et non la présence d'un public très féminin. Rarement dragués, les hôtes d'accueil sont en revanche souvent instigateurs de la drague, comme l'a montré l'observation à Entreprendre: envers les visiteuses, envers les hôtesses, mais pas envers les femmes vis-à-vis desquelles ils sont dans une position de subordination, ce qui confirme à nouveau les liens entre drague au travail et rapports de domination (15).

Ces liens entre drague au travail et position statutaire opèrent dès lors de manière inversée pour les hôtes et les hôtesses. La drague tend en effet à confirmer les hôtesses dans leur statut subordonné, tandis qu'elle permet aux hôtes une sortie du rôle.

Confrontées à la drague masculine, les hôtesses jouent parfois de ces interactions (c'est d'ailleurs ce qui est attendu d'elles), et peuvent se saisir de l'occasion pour affirmer elles aussi leur identité féminine dans sa dimension hétérosexuée et en retirer des gratifications. Les relations de drague les laissent toutefois relativement démunies, leur position professionnelle et leur rôle d'accueil - sourire, politesse et disponibilité - offrant peu de latitude pour éconduire les importuns et repousser des avances dont le caractère sérieux ou joué nécessite d'être finement jugé pour agir en conséquence. Se faire draguer fait implicitement partie du rôle professionnel des hôtesses, mais le fait que la sexualité soit officiellement bannie des lieux de travail implique également de faire respecter les limites de la bienséance, mission qui pèse avant tout sur les femmes, principales mises en cause dans une débauche qui adviendrait en raison de leur seule présence en milieu professionnel (Scotт, 1990). Ces injonctions contradictoires nécessitent alors pour les hôtesses de trouver ce que Pascale Molinier (2011) nomme, au sujet du sexuel dans le soin gériatrique, une "chimérique "bonne distance" », sans qu'aucun mode d'emploi leur soit fourni. S'extraire d'un rapport de drague peut en effet s'avérer ardu, lorsque le silence passe pour un encouragement et les paroles sèches pour un manquement aux obligations professionnelles, d'autant que les plus exposées à la drague - les plus subordonnées - sont aussi celles dont la position statutaire rend particulièrement délicate toute réaction de fermeté.

(15) Confirme également ces liens le fait que les hôtesses n'ont généralement aucune peine à s'extraire d'un rapport de drague qui leur déplairait avec un collègue hôte d'accueil, contrairement à ce qui se passe avec les visiteurs ou les autres salariés vis-à-vis desquels elles sont dans une position moins assurée (cf. infra).
À l'inverse, la drague peut être l'occasion pour les hôtes d'une sortie de leur position subordonnée, par la recréation d'un entre-soi masculin par-delà les statuts hiérarchiques, grâce à une connivence de genre avec les autres salariés hommes. En effet, l'observation à Entreprendre montre qu'outre les hôtesses, les salariés hommes draguent également les «extérieures», c'est-à-dire des femmes travaillant dans les quelques entreprises locataires de certains des étages d'Entreprendre, et amenées à ce titre à en fréquenter les locaux sans pour autant y être intégrées. Leurs tentatives de drague bénéficient de la complicité des hôtes d'accueil, qui occupent une position stratégique et peuvent être chargés de les prévenir par téléphone des moments où leurs cibles prennent des pauses; il leur arrive également de commenter a posteriori avec eux leurs techniques d'approche. Au cours des interactions se déroulant autour du hall d'accueil, se met donc en place entre hôtes et salariés masculins, sur un mode ludique, une validation réciproque de leurs compétences à la drague hétérosexuelle, et donc de leur virilité. Objets de transaction de la drague au travail, vecteurs de lien social plus qu'actrices, les femmes deviennent prétextes et enjeux d'une compétition masculine, qui permet aux hôtes de se mesurer aux autres salariés masculins et de sortir provisoirement de leur statut de subordonnés, tandis que les hôtesses se retrouvent pour leur part hors-jeu.

Ainsi, alors qu'en pénétrant dans un univers «féminin» les hôtes pourraient être menacés dans leur genre, il semble que la drague au travail - comme la recréation de tâches "féminines» et «masculines»- leur offre au contraire l'occasion de réaffirmer leur virilité, de rappeler l'«ordre hétérosexuel» et de mettre à distance la figure repoussoir du «pédé» (Clair, 2012). La drague leur permet aussi de nouer avec les salariés masculins de niveaux hiérarchiques supérieurs d'autres types de rapports, qui amènent à la question plus générale du rapport différentiel des hôtes et des hôtesses à l'encadrement et à leurs opportunités respectives de carrière.

\section{Carrières masculines et féminines dans l'accueil}

\section{Des hôtes qui sortent du lot}

Force est de constater qu'hôtes et hôtesses ne sont pas logés à la même enseigne dans les rapports avec leurs supérieur.e.s hiérarchiques, tout d'abord parce qu'ils ne sont pas perçus de la même manière. En effet, chez les prestataires d'accueil et à Entreprendre, l'idée selon laquelle des équipes d'accueil mixtes sont préférables car elles créent des ambiances plus «saines» est très répandue. La mixité est a minima vue comme productrice d'une ambiance plus sympathique, quand elle n'est pas 
associée à la discipline qu'apporteraient les hôtes dans un univers féminin que beaucoup imaginent propice aux disputes et aux rivalités. Comme le note Sabine ForTino (1999, p. 376), en milieu de travail féminin les hommes sont considérés comme des «agent [s] et facteur [s] de régulation sociale», et les hôtes d'accueil ne dérogent pas à la règle :

«Systématiquement on recrute des hommes. [...] Et puis l'hôte d'accueil, il peut aussi détendre l'équipe [...] quand les hôtesses ne s'entendent pas ou qu'elles sont en rivalité; ça peut être un plus. "

(Marie, 39 ans, assistante de direction et chargée du recrutement des hôte.sse.s d'accueil à Entreprendre, en poste depuis huit ans; entretien du 24 octobre 2011.)

«Moi, je préfère beaucoup plus travailler avec des hôtes que des hôtesses. Ils sont beaucoup plus faciles à gérer... [...] Les filles, elles vont tout de suite se regarder entre elles, tu vois, vraiment cet esprit de rivalité, j'ai remarqué...»

(Loretta, 22 ans, chef hôtesse en événementiel pour Réceptor, depuis un an; entretien du 8 novembre 2005.)

Si ces allusions aux rivalités entre hôtesses ravivent bien sûr des stéréotypes bien ancrés sur les femmes, elles peuvent aussi être lues comme un écho aux discours sur la "légèreté », qui caractériserait a contrario des hôtes d'accueil non captifs d'une performance de genre contraignante ( $c f$. supra). Par ailleurs, chez les prestataires en accueil en entreprise, les hôtesses sont aussi réputées être beaucoup moins fiables car plus enclines à l'absentéisme, alors même que la très faible proportion d'hôtes d'accueil rend pourtant toute généralisation de ce type fort hasardeuse :

«Alors ensuite, c'est une population quand même féminine, donc... Aussi c'est clair et net, on a beaucoup moins de problèmes avec les hôtes d'accueil. Les filles sont quand même beaucoup plus sensibles; donc petits problèmes de cour, de ceci, de cela ou de santé, on vient pas travailler... Et ça, c'est assez récurrent, voilà. Et ça, c'est vraiment lié au fait que c'est un métier vraiment féminin. »

(Samantha, 32 ans, responsable de l'accueil en entreprise pour Angélique Hôtesses, depuis quatre ans; entretien du 16 novembre 2005.)

Enfin, les désordres et l'inefficacité au travail provoqués par la présence d'une hôtesse, objet des convoitises masculines et des jalousies féminines, font également partie des topoï du secteur. Un magazine comme Arseg Info, revue professionnelle des directeurs des services généraux, qui sont, au sein des sociétés clientes, les interlocuteurs des sociétés prestataires d'accueil en entreprise, présente par exemple l'hôtesse idéale comme «jeune, mais pas trop belle pour ne pas provoquer d'embouteillages au bureau », et n'hésite pas à faire témoigner une société prestataire sur le fait que la beauté peut être «source de tracas» pour une hôtesse, à travers l'exemple d'une jeune femme «vraiment ravissante» ayant suscité la jalousie de l'ensemble du personnel féminin de la société cliente ${ }^{(16)}$.

Hôtes et hôtesses sont ainsi appréhendés à travers une grille de lecture genrée psychologisante de leurs supposées qualités. Ils ne bénéficient dès lors pas non plus de la même attention, tant de la part de l'encadrement que de celle des autres salariés qu'ils côtoient au quotidien. Chez les prestataires en accueil événementiel par exemple, où le recrutement est permanent et les effectifs très importants, les hôtes, minoritaires, sont souvent plus rapidement repérés et individualisés que les hôtesses. L'observation à Angélique Hôtesses a également montré que les responsables - des femmes - les trouvent généralement "délicieux» et que s'instaurent avec elles des rapports de badinage, voire de feinte séduction, comme le confirment également des entretiens effectués chez d'autres prestataires :

"Je suis très contente d'avoir un hôte. En plus, c'est un... [imitation ostensible d'un soupir de contentement], c'est un garçon délicieux, qui est très... là pour le coup, très... très poli, très courtois, très galant, très... oh c'est un amour! [rires]"

(Ariane, 26 ans, responsable de l'accueil en entreprise à Facil'Otess, depuis sept ans; entretien du 19 septembre 2005.)

À Entreprendre, la présence d'hommes à l'accueil ne passe pas non plus inaperçue et interroge beaucoup plus les autres salariés que celle de femmes. Il semble entendu qu'un poste d'accueil ne peut être que transitoire pour un homme, et les hôtes sont très régulièrement questionnés sur l'éventualité d'une mobilité professionnelle, se retrouvant presque sommés de se justifier d'occuper ce poste.

Ainsi, toutes les conditions semblent réunies pour que les hôtes bénéficient de carrières ascendantes au sein de l'accueil, voire pour qu'ils en sortent par le haut : des tâches qui les mènent à s'échapper physiquement des guichets, un rapport à la drague qui leur permet des sorties du rôle ponctuelles, des relations souvent privilégiées avec l'encadrement comme avec les autres salariés, souvent prompts à déceler en eux un potentiel exploitable hors de l'accueil. Qu'en est-il dans les faits ?

\section{Un "escalator de verre" pour les hôtes d'accueil ?}

La question de l'existence d'un «escalator de verre» pour les hôtes appelle une réponse nuancée, qui nécessite de distinguer l'accueil événementiel de l'accueil en entreprise, indépendamment du statut d'exercice - en prestation de services ou non.

Chez les prestataires, en accueil événementiel, on constate dans certaines sociétés que les hôtes

(16) Arseg Info, $\mathrm{n}^{\circ}$ 92, avril 2000; Arseg Info, $\mathrm{n}^{\circ}$ 71, mai 1998. 
Tableau 1 : Répartition des hôtes et des hôtesses en événementiel à Angélique Hôtesses selon leur âge et selon leur situation au moment de postuler

\begin{tabular}{|l|c|c|}
\hline \multicolumn{1}{|c|}{ Âge en 2006 } & $\begin{array}{c}\text { Accueil événementiel : hôtesses } \\
(\mathrm{NR}=0,5 \%, \mathrm{n}=245)\end{array}$ & $\begin{array}{c}\text { Accueil événementiel : hôtes } \\
(\mathrm{NR}=0 \%, \mathrm{n}=134)\end{array}$ \\
\hline Moins de 20 ans & $8 \%$ & $6 \%$ \\
\hline 20 à 24 ans & $51 \%$ & $39 \%$ \\
\hline 25 à 29 ans & $27 \%$ & $40 \%$ \\
\hline 30 ans et plus & $14 \%$ & $15 \%$ \\
\hline Total & $100 \%$ & $100 \%$ \\
\hline \multicolumn{1}{|c|}{ Situation au moment de postuler } & $\begin{array}{c}\text { Accueil événementiel : hôtesses } \\
(\mathrm{NR}=11 \%, \mathrm{n}=219)\end{array}$ & $\begin{array}{c}\text { Accueil événementiel : hôtes } \\
(\mathrm{NR}=14 \%, \mathrm{n}=115)\end{array}$ \\
\hline Recherche un emploi dans l'accueil & $3 \%$ & $0 \%$ \\
\hline Recherche un emploi dans une autre branche & $14 \%$ & $22 \%$ \\
\hline $\begin{array}{l}\text { Recherche un emploi complémentaire à une } \\
\text { activité principale }\end{array}$ & $15 \%$ & $23 \%$ \\
\hline Études en cours & $68 \%$ & $55 \%$ \\
\hline Total & $100 \%$ & $100 \%$ \\
\hline
\end{tabular}

Lecture : 8\% des hôtesses d'accueil événementiel inscrites à Angélique Hôtesses en 2006, avaient moins de 20 ans à cette date.

Champ : Échantillon par tirage aléatoire de 246 hôtesses et de 134 hôtes, parmi le personnel inscrit en accueil événementiel à Angélique Hôtesses en 2006.

Source : Base hôtesses événementiel, base hôtes événementiel (ScHüTz, 2011).

Tableau 2 : Comparaison de la répartition par diplôme des hôtes et des hôtesses en événementiel à Angélique Hôtesses et de la population française de 25 à 34 ans

\begin{tabular}{|l|c|c|c|c|}
\hline & \multicolumn{2}{|c|}{ Femmes } & \multicolumn{2}{c|}{ Hommes } \\
\hline \multirow{2}{*}{ Dernier diplôme obtenu } & $\begin{array}{c}\text { Hôtesses d'accueil } \\
\text { événementiel } \\
(\mathrm{NR}=9 \% ; \mathrm{n}=225)\end{array}$ & $\begin{array}{c}\text { Femmes de 25 à } \\
\mathbf{3 4} \text { ans, population } \\
\text { générale }\end{array}$ & $\begin{array}{c}\text { Hôtes d'accueil } \\
\text { événementiel } \\
(\mathrm{NR}=10 \%, \mathrm{n}=121)\end{array}$ & $\begin{array}{c}\text { Hommes de 25 à } \\
\mathbf{3 4} \text { ans, population } \\
\text { générale }\end{array}$ \\
\hline Inférieur au baccalauréat & $16 \%$ & $31,7 \%$ & $26 \%$ & $45,7 \%$ \\
\hline Baccalauréat & $39 \%$ & $22,6 \%$ & $37 \%$ & $19,7 \%$ \\
\hline Enseignement supérieur & $45 \%$ & $45,7 \%$ & $37 \%$ & $34,5 \%$ \\
\hline Total & $\mathbf{1 0 0 \%}$ & $\mathbf{1 0 0 \%}$ & $\mathbf{1 0 0 \%}$ & $\mathbf{1 0 0 \%}$ \\
\hline
\end{tabular}

Lecture : 16\% des hôtesses d'accueil événementiel inscrites à Angélique Hôtesses en 2006 avaient un diplôme inférieur au baccalauréat à cette date, tandis que c'était le cas de $31,7 \%$ des femmes de 25 à 34 ans en 2004.

Champ : Échantillon par tirage aléatoire de 246 hôtesses et de 134 hôtes, parmi le personnel inscrit en accueil événementiel à Angélique Hôtesses en 2006 / France métropolitaine, individus de 25 à 34 ans.

Source : Base hôtesses événementiel, base hôtes événementiel (ScHütz, 2011) / Insee, Données sociales : la société française - édition 2006, pp. 87-94.

se trouvent plus souvent que les hôtesses affectés sur des missions considérées comme prestigieuses, comme par exemple les défilés de mode, les spectacles ou les inaugurations. Les hommes sont réciproquement moins sollicités sur les conventions d'entreprises, congrès médicaux et stands de salons professionnels, qui emploient des hôtesses à profusion, et pour lesquels le tarif horaire appliqué est parfois plus faible. En cela, la distinction entre hôtes et hôtesses s'inscrirait dans une relative continuité avec la tradition du service domestique. Geneviève FraISSE (1979) distingue en effet la domesticité d'apparat, masculine, associée à un certain prestige social pour qui l'occupe, luxe voué à démontrer la fortune de ceux qui l'utilisent, du service domestique, féminin et dévalorisé socialement. Toutefois, chez tous les prestataires, le nombre de missions événementielles dédiées aux femmes est bien supérieur à celui où sont sollicités des hommes, et sont souvent aussi plus longues. S'il est ardu mais possible, pour une femme, de vivre exclusivement d'une activité d'hôtesse d'accueil en événementiel(17), c'est pour un hôte inenvisageable.

Si l'on compare par ailleurs le profil des hôtes et des hôtesses de l'accueil événementiel, tel qu'il a pu être établi à partir du travail statistique réalisé dans l'enquête sur les prestataires de services, on constate des différences qui rendent, elles aussi, la métaphore de l'escalator de verre peu ajustée à la situation (voir tableaux 1 et 2). En effet, alors que les hôtesses ont majoritairement moins de 24 ans, les hôtes ont fréquemment plus de 25 ans. Si les hôtesses sont plus diplômées que les hôtes d'accueil, on peut néanmoins faire la constatation suivante : alors qu'elles sont diplômées du supérieur dans une proportion équivalente à celle des

(17) On peut estimer entre 5 et $10 \%$ la proportion d'hôtesses - qui œuvrent généralement aussi comme chefs hôtesses faisant de l'accueil événementiel leur activité principale et parvenant à en vivre; celles-ci bénéficient généralement d'autres ressources, comme par exemple l'occupation d'un logement à titre gratuit (SCHÜTZ, 2011). 
femmes de leur génération, les hôtes d'accueil le sont pour leur part plus souvent que les hommes de leur génération : on peut alors les imaginer moins enclins encore que les hôtesses à occuper un emploi considéré comme non qualifié. Entrant plus tardivement dans l'accueil, les hommes semblent aussi y arriver un peu plus par défaut ${ }^{(18)}$. Ils sont moins souvent étudiants que les hôtesses et sont encore moins nombreux qu'elles à imaginer une carrière dans l'accueil $(0 \%$, contre $3 \%)$. Ils cherchent plus souvent qu'elles un emploi dans une autre branche(19), et sont plus souvent investis dans une activité principale (une fois sur deux une activité artistique), par rapport à laquelle l'accueil est vécu comme secondaire. Les hôtes d'accueil, globalement, semblent ainsi moins disposés à investir l'accueil dans le long terme, et lorsqu'ils le sont, c'est en tant qu'activité qui reste accessoire.

En accueil en entreprise, le très faible nombre d'hôtes d'accueil chez les prestataires rend difficile une comparaison statistique avec les hôtesses. Il apparaît néanmoins que les quelques hôtes rencontrés au cours de l'enquête ethnographique avaient tous obtenu un temps plein (ce qui est le cas de moins d'une hôtesse sur deux), et que l'un d'entre eux s'était même vu proposer de postuler sur un poste de responsable au siège, qu'il n'a finalement pas décroché. La situation observée à Entreprendre va également dans le sens de l'existence d'un escalator de verre pour les hommes à l'accueil; les histoires comparées de Christophe et de Bernadette, certes singulières, en illustrent néanmoins les mécanismes (voir encadré 2).

Tous deux dotés d'un semblable diplôme ${ }^{(20)}$, les deux «permanents» d'Entreprendre ont ainsi connu des évolutions de carrière fort différentes, l'un bénéficiant rapidement d'un temps plein puis d'une augmentation, tandis que l'autre se résignait au travail à temps partiel et à une stagnation

(18) En cela, les hôtes d'accueil ressemblent à la plupart des hommes exerçant des professions «féminines» et se distinguent des femmes exerçant des professions «masculines», pour qui ce choix est généralement le fruit d'un projet longuement mûri (GuichaRd-Claudic et al., 2008).

(19) Les hôtes ne sont pas les seuls hommes dans des métiers «féminins » à considérer leur activité comme transitoire. Les caissiers (BERNARD, 2011) parlent par exemple explicitement de quitter leurs fonctions à moyen terme, tandis que dans beaucoup d'autres cas, sans envisager de quitter à proprement parler leur secteur d'activité, les hommes se projettent dans des postes à responsabilité, ce qui aboutirait de fait à une profonde modification de la nature de leur activité (cf. entre autres Williams, 1992; J J 2008).

(20) Diplômes semblables mais de valeur différente, ayant été obtenus à vingt ans d'intervalle. Le contraste entre les destins professionnels de Bernadette et de Christophe n'en est donc que plus saisissant et ce, dès leur début de carrière puisque les diplômés de BTS dans les années 1980, comme Bernadette, étaient moins nombreux qu'aujourd'hui à être embauchés en tant que simples employés administratifs.

\section{Encadré 2 \\ Escalator de verre pour l'homme et carrière plafonnée pour la femme}

Christophe(1) et Bernadette(2) sont les deux «permanents» de l'accueil d'Entreprendre, qui compte également deux hôtesses et un hôte en «contrat étudiant». Bernadette a une cinquantaine d'années et travaille à Entreprendre depuis trente ans. Elle y a d'abord été hôtesse d'accueil après son BTS (brevet de technicien supérieur) en secrétariat, puis a travaillé quelques années à la comptabilité avant d'être réaffectée à l'accueil suite à une réorganisation. Elle est ainsi en poste à l'accueil depuis vingt-cinq ans et est encore aujourd'hui à temps partiel, quotité à $80 \%$ sous forme de deux demijournées non travaillées, qu'elle n'a pas choisie mais à laquelle elle a fini par se résigner du fait de la naissance de son enfant, il y a vingt-quatre ans, et de la montée en grade de son époux, devenu directeur d'un service dans une collectivité territoriale. Une première fois avant la naissance de son enfant, et plus récemment ces cinq dernières années, Bernadette a essayé d'être mutée à d'autres postes que l'accueil afin d'évoluer, mais n'y est pas parvenue.

Christophe a, quant à lui, une trentaine d'années et travaille à Entreprendre depuis cinq ans. Entré à temps partiel sur un contrat étudiant, alors qu'il tentait de valider une troisième année d'un cursus d'économie après un BTS d'assistant de PME-PMI, il finit par abandonner ses études. Il se voit proposer au bout de trois ans un temps plein en CDD, qu'il accepte, faute d'autres perspectives. Deux ans plus tard, son emploi est transformé en CDI, toujours à temps plein, avec pour condition qu'il reprenne une partie des tâches de l'«homme à tout faire» parti en retraite. Les fonctions de Christophe s'étendent alors et le mènent à travailler beaucoup plus souvent en dehors de l'accueil, auquel il ne consacre plus que $50 \%$ de son temps - il sert par exemple occasionnellement de chauffeur au directeur. II s'occupe également des tâches relevant plutôt des services généraux - entretien des véhicules de service, gestion des stocks alimentaires, des bonbonnes d'eau, des petits travaux du bâtiment comme le changement d'ampoules, organisation technique des assemblées générales, etc. Depuis son changement de statut, Christophe n'est plus questionné par les autres salariés sur ses ambitions professionnelles : on considère qu'il a dorénavant plus de responsabilités; en toute logique, il a d'ailleurs reçu une augmentation. Son retrait de l'accueil alourdit néanmoins le travail du reste des intervenant.e.s, qui traitent en effectif réduit autant d'appels et de tâches administratives qu'auparavant.

Bernadette souligne pour sa part qu'elle n'a jamais été augmentée en plus de trente ans passés à Entreprendre.

(1) Fils de parents ouvriers, licenciés suite à la fermeture de leur usine - le père n'a pas retrouvé d'emploi, la mère est devenue femme de ménage; entretien du 4 novembre 2011.

(2) Fille d'une mère au foyer et d'un père entrepreneur indépendant, elle a été élevée par sa grand-mère retraitée; entretien informel du 14 octobre 2011. 
salariale. Ce récit donne à voir la force du lien entre genre et forme d'emploi, qui fait du temps partiel un temps de travail "féminin» que l'on n'envisage pas d'imposer à un homme (ANGElOFF, 2000), et permet aussi de montrer en action les ressorts de la différenciation des carrières féminines et masculines précédemment mis en évidence. C'est en effet parce que, pour aucun des autres salariés, Christophe ne semble «à sa place» à l'accueil, que ses tâches sont redéfinies en un sens plus «masculin». C'est bien aussi parce que Christophe s'éloigne de plus en plus de l'accueil que ses tâches sont dorénavant perçues comme plus «techniques» et impliquant plus de responsabilités, ce qui justifie son augmentation de salaire. Ainsi, non seulement Christophe bénéficie-t-il d'une promotion (sociale et salariale) alors que Bernadette n'en a jamais reçu mais en plus, les conditions de travail de cette dernière se détériorent à la suite de cette promotion. Il semble donc que si la métaphore de l'escalator de verre s'avère peu pertinente pour l'accueil événementiel, où les hôtes ont moins d'opportunités d'emploi et ont un profil un peu différent de celui des hôtesses, qui les dispose moins qu'elles encore à s'investir sur le long terme, celle-ci semble pleinement fonctionner pour l'accueil en entreprise.

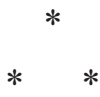

Le travail pionnier de Christine Williams (1992) rend compte des avantages structurels dont bénéficient les hommes exerçant des activités pensées comme "féminines», et qui contrastent avec le sort réservé aux femmes dans des situations symétriques. Les hommes qu'elle étudie ne rencontrent pas de frein à leur embauche et sont souvent au contraire très bien accueillis. Ils sont canalisés, parfois malgré eux, vers les tâches les plus valorisées de l'activité, qui permettent une évolution, en particulier les tâches d'administration moins en contact direct avec le public. Leur inscription dans des réseaux de sociabilité informelle avec leurs supérieur.e.s hiérarchiques accélère leur progression de carrière. Ces hommes recherchent parfois activement ces promotions pour contrer le stigmate de l'identification à une profession "féminine», souvent dévalorisée et mal rémunérée, et répondre aux défis posés à leur masculinité, mécanisme qui se cumule aux autres pour les aspirer vers le haut. D'autres auteures ont mis l'accent sur le fait que ces hommes sont objectivement plus disponibles pour l'activité professionnelle que leurs homologues féminines, en raison du maintien de la division sexuelle du travail domestique et familial dans la sphère privée (Le Feuvre, Laufer, 2008). Quoi qu'il en soit, il apparaît que choisir un métier atypique n'aboutit en aucun cas pour ces hommes à une "féminisation", puisqu'ils se voient reconnaître l'exclusivité d'un certain nombre de capacités ou compétences habituellement pensées comme masculines, si bien qu'ils occupent une position favorable dans la division sexuelle du travail qui se joue dans ces métiers (Le Feuvre, Laufer, 2008).

Notre étude s'inscrit dans la continuité de ces analyses, qu'elle confirme amplement. Elle met en outre en évidence la façon dont les conceptions genrées du corps, et non des seules compétences «masculines» et "féminines», participent elles aussi à la mise en place d'une division sexuelle des tâches dans un métier "féminin», assurant aux hommes plus de marges de manœuvre et une plus grande liberté. À travers une analyse de la "drague », elle pointe le rôle de la sexualité dans les organisations sur le maintien de la subordination et d'un certain isolement des femmes au travail, mais souligne aussi ses vertus d'intégration des hommes dans un collectif masculin transstatutaire. Alors même que le personnel d'accueil occupe une position à la marge, voire extérieure, dans la division du travail (souvent traduite spatialement par l'occupation de sas et de halls d'entrée) si ce n'est dans celle de l'emploi (via le statut de prestataire), les hôtes parviennent à s'extraire de cette marginalité, contrairement aux hôtesses et en dépit de leur position «minoritaire». Frappe ici la force de ce mécanisme d'inclusion des hommes dans un collectif organisationnel, qui, par-delà l'accueil, a été mis en évidence dans d'autres situations et ce, sans que la sexualité intervienne ${ }^{(21)}$. En définitive, on peut s'interroger sur la notion même de position «minoritaire», puisque les hôtes d'accueil peuvent comme d'autres hommes être «minoritaires » au sein de leur fonction mais ne le sont ni dans leur position de genre, qui exerce une force de rappel, ni au sein des organisations, où ils côtoient quotidiennement d'autres hommes avec lesquels peut jouer une certaine solidarité.

(21) Marine Cordier (2007) montre, par exemple, que les circassiennes tendent plus à s'investir dans des numéros en solo, tandis que les circassiens appartiennent plus souvent à des collectifs créatifs où ils sont auteurs et metteurs en scène. Ces collectifs donnent une impulsion à leur carrière et excluent d'autant plus efficacement les femmes qu'ils résultent de liens d'amitié et d'affinités électives, qui ne sont pas pensés comme une défense volontaire contre l'arrivée de femmes. ForTino (2002, p. 128) expose pour sa part comment, dans une entreprise publique où la progression de carrière se fait surtout par cooptation, les hommes se sentent membres d'un collectif et vivent leurs (fréquentes) promotions comme s'inscrivant dans un parcours naturel et légitime, alors que les femmes vivent leurs (rares) promotions comme des faveurs accidentelles et des situations uniques, non généralisables et encore moins exemplaires, dont elles doivent être reconnaissantes. 


\section{Bibliographie}

Angeloff T. (2000), Le temps partiel: un marché de dupes?, Paris, Syros.

Angeloff T., Arborio A.-M. (2002), «Des hommes dans des "métiers de femmes": mixité au travail et espaces professionnels dévalorisés», Sociologia del lavoro, $\mathrm{n}^{\circ} 85$, pp. 123-135.

Baudoux C., Zaidman C. (1992), Égalité entre les sexes, mixité et démocratie, Paris, L'Harmattan.

Bauvet S. (2010), «Quelques économies des relations au travail dans la sécurité privée», communication au colloque La dimension relationnelle des métiers de service: cache-sexe ou révélateur de genre? (2-4 septembre 2010), Université de Lausanne.

Bernard S. (2011), "“C'est pas un homme qui va travailler dans les dentelles!" De l'impossible mixité dans la grande distribution ?», in Cacouault-Bitaud M., Charles F. (dir.), Quelle mixité dans les formations et les groupes professionnels? Enquêtes sur les frontières et le mélange des genres, Paris, L'Harmattan, pp. 35-55.

Bessin M. (2008), «Les hommes dans le travail social : le déni du genre», in Guichard-Claudic Y., Kergoat D., Vilbrod A. (dir.), L'inversion du genre. Quand les métiers masculins se conjuguent au féminin ... et réciproquement, Rennes, Presses universitaires de Rennes, pp. 357-370.

Bodin R. (2011), «De la division sexuée des postes dans l'éducation spécialisée», in Cacouault-Bitaud M., Charles F. (dir.), Quelle mixité dans les formations et les groupes professionnels? Enquêtes sur les frontières et le mélange des genres, Paris, L'Harmattan, pp. 75-86.

Brochier C. (2011), «Le monde social d'une petite usine», Revue française de sociologie, vol. 52, $\mathrm{n}^{\circ} 1$, pp. 3-36.

Buscatto M., Marry C. (2009), «Le plafond de verre dans tous ses éclats. La féminisation des professions supérieures au Xx ${ }^{\mathrm{e}}$ siècle», Sociologie du travail, vol. 51, $\mathrm{n}^{\circ} 2$, pp. 170-182.

Buscatto M., Fusulier B. (2013), «Les "masculinités" à l'épreuve des métiers "féminins"», Recherches sociologiques et anthropologiques, vol. 44, n², pp. 1-19.

Cacouault-Bitaud M., Charles F. (dir.) (2011), Quelle mixité dans les formations et les groupes professionnels? Enquêtes sur les frontières et le mélange des genres, Paris, L'Harmattan.

Charrier P. (2004), «Comment envisage-t-on d'être sage-femme quand on est un homme ?», Travail, genre et sociétés, $\mathrm{n}^{\circ} 12$, pp. 105-124.

Charrier P. (2008), «Des hommes dans une profession "traditionnellement" féminine: choix professionnel et dénomination chez les hommes sages-femmes», in Guichard-Claudic Y., Kergoat D., Vilbrod A. (dir.), L'inversion du genre. Quand les métiers masculins se conjuguent au féminin... et réciproquement, Rennes, Presses universitaires de Rennes, pp. 231-241.
Clair I. (2012), «Le pédé, la pute et l'ordre hétérosexuel», Agora, n 60, pp. 67-78.

Connell R. W. (1995), Masculinities, Cambridge, Polity Press.

Connell R. W., Messerschmidt J. W. (2005), " Hegemonic masculinity : rethinking the concept ", Gender and society, vol. 19, n 6, pp. 829-859.

Cordier M. (2007), «Corps en suspens: les genres à l'épreuve dans le cirque contemporain», in GuichardClaudic Y., Kergoat D. (dir.), «Inversion du genre. Corps au travail et travail des corps"», Les cahiers du genre, $\mathrm{n}^{\circ}$ 42, pp. 79-100.

Cresson G. (2010), «Indicible mais omniprésent: le genre dans les lieux d'accueil de la petite enfance», Les cahiers du genre, $\mathrm{n}^{\circ} 49$, pp. 15-33.

Cromer S., Lemaire D. (2007), «L'affrontement des sexes en milieu de travail non mixte, observation du système de genre», in Guichard-Claudic Y., Kergoat D. (dir.), «Inversion du genre. Corps au travail et travail des corps », Les cahiers du genre, $\mathrm{n}^{\circ} 42$, pp. 61-78.

Cross S., Bagilhole B. (2002), « Girls' jobs for the boys? Men, masculinity and non-traditional occupations "), Gender, work and organization, vol. 9, n² 2, pp. 204-226.

Douguet F., Vilbrod A. (2008), «Les infirmiers libéraux : singularité des trajectoires professionnelles et des pratiques de soin », in Guichard-Claudic Y., Kergoat D., Vilbrod A. (dir.), L'inversion du genre. Quand les métiers masculins se conjuguent au féminin... et réciproquement, Rennes, Presses universitaires de Rennes, pp. 273-282.

ForTino S. (1999), «De la ségrégation sexuelle des postes à la mixité au travail : étude d'un processus », Sociologie du travail, vol. 41, n 4, pp. 363-384.

Fortino S. (2002), La mixité au travail, Paris, La Dispute.

Fraisse G. (1979), Femmes toutes mains : essai sur le service domestique, Paris, Seuil.

Galbraith M. (1992), « Understanding career choices of men in elementary education ", Journal of educational research, vol. $85, \mathrm{n}^{\circ} 4$, pp. 246-253.

Gollac M., Volkoff S. (2002), «La mise au travail des stéréotypes de genre: les conditions de travail des ouvrières », Travail, genre et sociétés, $n^{\circ} 8$, pp. 25-53.

Guichard-Claudic Y., Kergoat D., Vilbrod A. (dir.) (2008), L'inversion du genre. Quand les métiers masculins se conjuguent au féminin... et réciproquement, Rennes, Presses universitaires de Rennes.

Ghigi R. (2004), «Le corps féminin entre science et culpabilisation. Autour d'une histoire de la cellulite», Travail, genre et sociétés, $\mathrm{n}^{\circ} 12$, pp. 55-75.

Guillaume C., Pochic S. (2007), «La fabrication organisationnelle des dirigeants. Un regard sur le plafond de verre», Travail, genre et sociétés, n 17, pp. 79-101. 
Guillaumin C. (1981), «Femmes et théories de la société : remarques sur les effets théoriques de la colère des opprimées », Sociologie et société, n 2, pp. 19-32.

Guillaumin C. (1992), Sexe, race et pratique du pouvoir, Indigo et Côté-Femmes.

Gutek B. A. (1995), The dynamics of service, San Francisco, Jossey-Bass Publishers.

Henson K. D., Rogers J. K. (2001), " "Why Marcia you've changed!" Male clerical temporary workers doing masculinity in a feminized occupation », Gender and society, vol. 15, $\mathrm{n}^{\circ} 2$, pp. 218-238.

Herman E. (2007), «La bonne distance. L'idéologie de la complémentarité légitimée en centre de loisirs», in Guichard-Claudic Y., Kergoat D. (dir.), «Inversion du genre. Corps au travail et travail des corps », Les cahiers du genre, $\mathrm{n}^{\circ} 42$, pp. 121-139.

Hidri Neys O. (2013), «Le jeu des apparences : piège ou profit ? Enquête auprès des hôtesses d'accueil et de caisse de la distribution sportive», Travail et emploi, $\mathrm{n}^{\circ} 134$, pp. 75-89.

JABOIN Y. (2008), «La construction de l'identité professionnelle masculine dans un secteur en voie de mixité : le cas des hommes enseignant à l'école maternelle», in Guichard-Claudic Y., Kergoat D., Vilbrod A. (dir.), L'inversion du genre. Quand les métiers masculins se conjuguent au féminin ... et réciproquement, Rennes, Presses universitaires de Rennes, pp. 243-255.

Kergont D. (1992), «La gestion de la mixité dans un mouvement social : le cas de la coordination infirmière», in Baudoux C., Zaidman C. (dir.), Égalité entre les sexes, mixité et démocratie, Paris, L'Harmattan, pp. 261-278.

Kergoat D. (2000), «Division sexuelle du travail et rapports de sexe», in Hirata H., Laborie F., Le Doaré H., Senotier D. (coord.), Dictionnaire critique du féminisme, Paris, Presses universitaires de France, pp. 35-44.

LADA E. (2008), «Le genre en pratique et pratiques du genre des hommes dans des emplois de service dits "de femme" ", in Guichard-Claudic Y., Kergoat D., Vilbrod A. (dir.), L'inversion du genre. Quand les métiers masculins se conjuguent au féminin... et réciproquement, Rennes, Presses universitaires de Rennes, pp. 371-383.

LAUfer J. (1982), La féminité neutralisée ? Les femmes cadres dans l'entreprise, Paris, Flammarion.

LAUFER J. (2005), «La construction du plafond de verre : le cas des femmes cadres à potentiel», Travail et emploi, $\mathrm{n}^{\circ} 102$, pp. 31-44.

Le Feuvre N., Laufer J. (2008), «Quand l'avancée en mixité est le fait d'hommes. Introduction», in GuichardClaudic Y., Kergoat D., Vilbrod A. (dir.), L'inversion du genre. Quand les métiers masculins se conjuguent au féminin... et réciproquement, Rennes, Presses universitaires de Rennes, pp. 207-213.
MACKInNON C. A. (1979), Sexual harassment of working women : a case of sex discrimination, New Haven, Yale University Press.

Molinier P. (2011), «Le sexuel dans le soin gériatrique. Une "difficulté dans la réalité"》, Genre, sexualité \& société [En ligne], $\mathrm{n}^{\circ}$ 6; disponible à l'adresse : http://gss.revues. org/index2193.html; consultée le 18 décembre 2014.

Murcier N. (2008), «Petite enfance et rapports sociaux de sexe: la formation des professionnel(le)s de la petite enfance, idéologies et représentations sociales », in Guichard-Claudic Y., Kergoat D., Vilbrod A. (dir.), L'inversion $d u$ genre. Quand les métiers masculins se conjuguent au féminin... et réciproquement, Paris, Presses universitaires de Rennes, pp. 215-230.

Peretz H. (1992), «Le vendeur, la vendeuse et leur cliente. Ethnographie du prêt-à-porter de luxe», Revue française de sociologie, vol. 33, $\mathrm{n}^{\circ}$ 1, pp. 49-72.

Perrot M. (1987), «Qu'est-ce qu'un métier de femme?», Le Mouvement social, $\mathrm{n}^{\circ} 140, \mathrm{pp} .2-10$.

Philippe C. (2008), «Le conseil conjugal et familial au masculin », in Guichard-Claudic Y., Kergoat D., Vilbrod A. (dir.), L'inversion du genre. Quand les métiers masculins se conjuguent au féminin... et réciproquement, Rennes, Presses universitaires de Rennes, pp. 257-271.

Picot G. (2011), «Usage du genre et mixité au travail dans les services de soins hospitaliers », in CacouaultBitaud M., Charles F. (dir.), Quelle mixité dans les formations et les groupes professionnels? Enquêtes sur les frontières et le mélange des genres, Paris, L'Harmattan, pp. 87-101.

Scotт J. W. (1990), «L'ouvrière, mot impie, sordide», Actes de la recherche en sciences sociales, $\mathrm{n}^{\circ} 83$, pp. 2-15.

SchüTz G. (2006), «Hôtesse d'accueil, les attendus d'un "petit boulot" féminin pour classes moyennes», Terrains et travaux, $\mathrm{n}^{\circ} 10$, pp. 137-156.

Schütz G. (2011), Travail et relations hiérarchiques en contexte d'externalisation. Les prestations de services d'hôtesses d'accueil, Thèse de doctorat en sociologie, Paris, EHESS.

Sorignet P.-E. (2004), «La construction des identités sexuées et sexuelles au regard de la socialisation professionnelle : le cas des danseurs contemporains », Sociologie de l'art, OPuS 5, pp. 9-34.

Williams C. L. (1992), « The glass escalator : hidden advantages for men in the "female" professions ", Social problems, vol. 39, $\mathrm{n}^{\circ} 3$, pp. 253-267.

Zolesio E. (2009), "“Chirurchiennes" de garde et "humour chirurchical". Posture féminine de surenchère dans l'humour sexuel et scatologique», Interrogations, [En ligne], $\mathrm{n}^{\circ} 8$; disponible en ligne à l'adresse : http:// www.revue-interrogations.org/Chirurchiennes-de-gardeet-humour; consultée le 18 décembre 2014. 


\section{Annexe}

Tableau : Caractéristiques des enquêtés

\begin{tabular}{|c|c|c|c|c|c|}
\hline Enquêté & Âge & $\begin{array}{c}\text { Fonction occupée } \\
\text { et ancienneté dans la } \\
\text { fonction ou l'entreprise }\end{array}$ & Entreprise & $\begin{array}{l}\text { Histoire } \\
\text { familiale }\end{array}$ & $\begin{array}{c}\text { Trajectoire sociale } \\
\text { et professionnelle } \\
\text { antérieure }\end{array}$ \\
\hline Amina & 25 ans & $\begin{array}{l}\text { Chef hôtesse événemen- } \\
\text { tiel depuis } 6 \text { ans. }\end{array}$ & Réceptor & $\begin{array}{l}\text { Algérienne, fille d'une mère } \\
\text { au foyer et d'un homme } \\
\text { cadre dirigeant d'une grande } \\
\text { société. }\end{array}$ & $\begin{array}{l}\text { Amina s'est installée en France } \\
\text { pour ses études d'architecture, } \\
\text { qu'elle est en train d'achever. }\end{array}$ \\
\hline Ariane & 26 ans & $\begin{array}{c}\text { Responsable de l'accueil } \\
\text { en entreprise depuis } \\
7 \text { ans. }\end{array}$ & Facil'Otess & $\begin{array}{l}\text { Fille d'une secrétaire et d'un } \\
\text { homme décédé. }\end{array}$ & $\begin{array}{l}\text { Entrée dans l'accueil (ancienne } \\
\text { hôtesse d'accueil en entreprise) } \\
\text { après un échec au baccalauréat, } \\
\text { qu'elle obtiendra plus tard en } \\
\text { candidate libre. }\end{array}$ \\
\hline Bernadette & $\begin{array}{c}50 \text { ans } \\
\text { (environ) }\end{array}$ & $\begin{array}{l}\text { Hôtesse d'accueil à } \\
\text { temps partiel depuis } \\
25 \text { ans. }\end{array}$ & Entreprendre & $\begin{array}{l}\text { Fille d'une mère au foyer et } \\
\text { d'un père entrepreneur indé- } \\
\text { pendant, a été élevée par sa } \\
\text { grand-mère retraitée. }\end{array}$ & $\begin{array}{l}\text { BTS secrétariat, poste à l'accueil, } \\
\text { puis à la comptabilité, puis } \\
\text { de nouveau à l'accueil. Toute } \\
\text { sa carrière s'est déroulée à } \\
\text { Entreprendre (voir encadré 2). }\end{array}$ \\
\hline Christophe & 30 ans & $\begin{array}{l}\text { Hôte d'accueil à temps } \\
\text { plein depuis } 5 \text { ans. }\end{array}$ & Entreprendre & $\begin{array}{l}\text { Fils de parents ouvriers, } \\
\text { licenciés suite à la fermeture } \\
\text { de leur usine - le père n'a } \\
\text { pas retrouvé d'emploi, la } \\
\text { mère est devenue femme de } \\
\text { ménage. }\end{array}$ & $\begin{array}{l}\text { BTS d'assistant de PME-PMI. } \\
\text { Entre à Entreprendre en contrat } \\
\text { étudiant à temps partiel avant } \\
\text { de passer en CDD à temps plein } \\
\text { au bout de trois ans, puis en } \\
\text { CDI deux ans plus tard (voir } \\
\text { encadré 2). }\end{array}$ \\
\hline Laurence & 39 ans & $\begin{array}{l}\text { Chargée de mission } \\
\text { depuis } 19 \text { ans. }\end{array}$ & Entreprendre & $\begin{array}{l}\text { Fille d'un père directeur } \\
\text { d'un service en collectivité } \\
\text { territoriale et d'une mère au } \\
\text { foyer. }\end{array}$ & $\begin{array}{l}\text { Ancienne hôtesse d'accueil en } \\
\text { contrat étudiant pendant trois ans, } \\
\text { lors de ses études de droit. }\end{array}$ \\
\hline Loretta & 22 ans & $\begin{array}{l}\text { Chef hôtesse en événe- } \\
\text { mentiel depuis } 1 \text { an. }\end{array}$ & Réceptor & $\begin{array}{l}\text { Fille d'un chauffeur de } \\
\text { taxi et d'une secrétaire de } \\
\text { direction. }\end{array}$ & $\begin{array}{l}\text { Se consacre pour l'instant pleine- } \\
\text { ment à l'accueil après des études } \\
\text { de langue à l'université. }\end{array}$ \\
\hline Marc & 36 ans & $\begin{array}{l}\text { Hôte à temps plein } \\
\text { depuis } 6 \text { ans. }\end{array}$ & $\begin{array}{c}\text { Business } \\
\text { Accueil }\end{array}$ & $\begin{array}{l}\text { Fils d'un petit entrepreneur } \\
\text { et d'une mère au foyer. }\end{array}$ & $\begin{array}{l}\text { Se définit comme musicien et, } \\
\text { ne parvenant pas à vivre de cette } \\
\text { activité, a toujours exercé ce qu'il } \\
\text { appelle des «petits boulots». }\end{array}$ \\
\hline Marie & 39 ans & $\begin{array}{c}\text { Assistante de direction; } \\
\text { chargée du recrutement } \\
\text { des hôte.sse.s d'accueil } \\
\text { depuis } 8 \text { ans. }\end{array}$ & Entreprendre & Information non connue & $\begin{array}{l}\text { C'est son troisième poste } \\
\text { d'assistante de direction. Elle } \\
\text { a auparavant travaillé dans une } \\
\text { entreprise de textile puis un } \\
\text { organisme de formation, après un } \\
\text { BTS assistante de direction et une } \\
\text { licence en langues et civilisations } \\
\text { étrangères. }\end{array}$ \\
\hline Nicolas & 25 ans & $\begin{array}{l}\text { Hôte d'accueil en événe- } \\
\text { mentiel depuis } 4 \text { ans et } \\
\text { demi. }\end{array}$ & Bienvenue & $\begin{array}{l}\text { Fils d'un chef de petite } \\
\text { entreprise et d'une femme } \\
\text { travaillant à domicile pour } \\
\text { une société de sténodactylo. }\end{array}$ & $\begin{array}{l}\text { Se consacre à la musique après } \\
\text { des études en ingénierie du son } \\
\text { et un premier emploi dans cette } \\
\text { branche. }\end{array}$ \\
\hline Pauline & 22 ans & $\begin{array}{l}\text { Hôtesse d'accueil à } \\
\text { temps partiel depuis } \\
2 \text { ans et demi. }\end{array}$ & Entreprendre & $\begin{array}{l}\text { Sa mère est cadre adminis- } \\
\text { trative dans une entreprise } \\
\text { postale; son père, entre- } \\
\text { preneur indépendant à } \\
\text { l'étranger. }\end{array}$ & $\begin{array}{l}\text { C'est son premier emploi alors } \\
\text { qu'elle débute des études de } \\
\text { philosophie, qu'elle abandonne un } \\
\text { mois après la rentrée. }\end{array}$ \\
\hline Samantha & 32 ans & $\begin{array}{c}\text { Responsable de l'accueil } \\
\text { en entreprise depuis } \\
4 \text { ans. }\end{array}$ & $\begin{array}{l}\text { Angélique } \\
\text { Hôtesses }\end{array}$ & $\begin{array}{l}\text { Son père est PDG d'une } \\
\text { concession automobile, } \\
\text { sa mère dirige un salon de } \\
\text { coiffure. }\end{array}$ & $\begin{array}{l}\text { A eu une expérience de quelques } \\
\text { années au siège d'une agence } \\
\text { d'intérim comme assistante, après } \\
\text { une maîtrise d'administration } \\
\text { des entreprises et société (AES) } \\
\text { faisant suite à un BTS action } \\
\text { commerciale. }\end{array}$ \\
\hline Steve & 22 ans & $\begin{array}{l}\text { Hôte d'accueil en événe- } \\
\text { mentiel depuis un an et } \\
\text { demi. }\end{array}$ & Biепvепие & $\begin{array}{l}\text { Fils d'une hôtesse de l'air et } \\
\text { d'un homme d'affaires. }\end{array}$ & $\begin{array}{l}\text { Étudie le chant lyrique dans un } \\
\text { conservatoire municipal. }\end{array}$ \\
\hline
\end{tabular}

